Психология. Журнал Высшей школы экономики,

2020. T. 17. № 4. C. 645-657. DOI: 10.17323/1813-8918-2020-4-645-657

\title{
РОЛЬ МОТОРНОГО КОМПОНЕНТА В ПРОЦЕССЕ РЕШЕНИЯ ИНСАЙТНЫХ ЗАДАЧ
}

\author{
А.А. КУРИЦЫН , А.В. ЧИСТОПОЛЬСКАЯ
}

\begin{abstract}
а Ярославский государственньй университет им. П.Г. Демидова, 150000, Россия, Ярославль, ул. Советская, Ә. 14
\end{abstract}

\begin{abstract}
Резюме
В когнитивной психологии сегодня достаточно актуальны идеи влияния тела и моторики на психические процессы. Совокупность этих идей формирует новое направление исследований - программу исследований Embodied cognition (Воплощенное познание). Однако, несмотря на широкую распространенность, только небольшое количество исследований применяет положения этого подхода при изучении феномена инсайта. На данный момент существует несколько теорий, которые по-разному пытаются объяснить механизмы инсайтного решения: неспецифический подход, который отрицает различия в процессе решения инсайтных и неинсайтных задач, и специфический подход, который предполагает наличие специальных механизмов решения инсайтных задач. Данное исследование, опираясь на идеи подхода воплощенного познания и концепцию специфического подхода к пониманию инсайта, рассматривает процессы решения инсайтных задач в зависимости от моторной активности, в различной степени содержащей принципы решения задачи на симметрию: принцип декомпозиции чанка и принцип симметрии. Были сформированы 3 экспериментальные группы моторной активности, которые по-разному воплощают оба принципа решения задачи. Различия обнаружены во времени решения в зависимости от сопутствующего типа моторной активности. Обнаружено фасилитирующее влияние моторной активности, воплощающей оба основных принципа решения инсайтной задачи. Предполагается, что наличие в моторной активности принципов решения задачи имплицитно запускает механизмы смены репрезентации. Намечены планы будущего исследования, в котором предполагается проверка принципов решения инсайтной задачи на основе безынструментальной моторной активности. Полученные результаты согласуются с идеями Я.А. Пономарева о роли побочного продукта в мыслительных процессах.
\end{abstract}

Ключевые слова: инсайт, моторная активность, воплощенное познание, декомпозиция чанка, задача на симметрию.

\section{Воплощенное познание и изучение инсайта}

В отечественной психологии исследования творческого мышления особое место занимают работы Я.А. Пономарева. Автор не только разработал общую концепцию творчества, методологические принципы исследования, но и оставил богатое эмпирическое наследие. В частности, актуальными и сегодня

Исследование выполнено в рамках проекта МК-70.2019.6. 
остаются идеи побочного продукта, которые находят отражение в концепции воплощенного познания в когнитивной психологии. Экспериментально эти идеи были проверены на материале предварительного выполнения задания с политипной панелью и последующим решением задачи на прохождение лабиринта. Задача на прохождение лабиринта достаточно сложная и, как правило, не решается за отведенное в эксперименте время. Предварительно испытуемый выполнял задание на расположение планок на панели определенным образом в соответствии с инструкциями. Далее испытуемому надо было пройти лабиринт, оптимальный путь по которому точно копирует порядок и форму итогового расположения планок в предшествующем задании. В результате данного эксперимента было получено существенное сокращение проб в прохождении лабиринта (почти в 10 раз: с 70-80 до 8-10). Однако при повороте панели с планками на $180^{\circ}$ и сохранной пространственной ориентации лабиринта, выполнении промежуточного нейтрального задания или увеличении промежутка времени между заданиями фасилитирующий эффект предварительного задания исчезает. Я.А. Пономарев делает на основании этих результатов вывод о влиянии побочного продукта на мыслительные процессы (Пономарев, 1976).

В контексте идеи воплощенного познания можно говорить о том, что в данном эксперименте у испытуемого сформировалась моторная программа, воплотился моторный принцип решения сложной задачи на прохождение лабиринта, а любые помехи в актуализации данной программы (пространственные, временны́е, интерферирующие) приводят к ухудшению решения задачи. Как можно заметить, эти классические идеи побочного продукта достаточно актуальны в рамках концепции воплощенного познания.

Воплощенное познание (embodied cognition, далее сокращенно ВП) - это целая совокупность походов и направлений в когнитивистике для рассмотрения человеческого тела и его активности как одной из наиболее важных детерминант, обуславливающих психические процессы человека. Направление берет свое начало из критики информационного подхода, а именно компьютерной метафоры как объяснительной модели человеческого познания, тем самым возвращая «человека» в когнитивную психологию. Существует несколько теоретических обзоров ВП, в которых рассматриваются особенности и внутренняя организация направления ВП (Уилсон, 2012; Glenberg, 2010). Так, стоит отметить работу В.Ф. Спиридонова и Н.И. Логинова (Логинов, Спиридонов, 2017a, 2017б), где подробно рассмотрена одна из возможных классификаций.

Особое внимание уделяется влиянию моторной активности на высокоуровневые психические процессы, т.е. процессы, которые детерминированы в большей степени характеристиками субъекта и осуществляют переработку информации на основе опыта (Чистопольская и др., 2019). Так, например, широко представлены исследования, которые рассматривают строение и положение человеческого тела в пространстве как базис для построения языковой системы человека (Лакофф, Джонсон, 2004), причем в случае билингвов оба языка задействуют сенсомоторный опыт одинаково для слов, описывающих эмоции, и слов с конкретной пространственной характеристикой 
(Dudschig et al., 2014). Моторная активность, в свою очередь, может влиять на выбор стратегии решения для модифицированных задач Лачинса (Werner, Raab, 2013, 2014; Werner et al., 2019). Достаточно широкую применимость принципы ВП нашли в исследованиях, посвященных практическому решению арифметических задач. Так, Н. Мишо и др. (Michaux et al., 2013) было проверено предположение о том, что счет и решение арифметических задач онтологически связаны между собой. Было обнаружено, что если при выполнении простейших операций счета в уме (сложение, вычитание) занять пальцы руки испытуемого каким-либо простым моторным упражнением, то успешность решения таких задач падает.

Однако не для всех областей когнитивной науки характерно широкое применение концепций подхода ВП, например, для изучения феномена инсайта. Инсайт - ключевой момент в процессе решения, связанный со скачкообразным переструктурированием проблемного поля, которое приводит к нахождению ответа (не обязательно верного) и часто сопровождается яркими переживаниями (Спиридонов, 2006). Сам феномен инсайта поддается регистрации, однако механизмы его возникновения остаются спорными. Можно выделить два доминирующих подхода для поиска ответа на этот вопрос: специфический (неогештальтистский) и неспецифический.

Сторонники неспецифического подхода, развивая идеи А. Ньюэла и Г. Саймона (Newell, Simon, 1972) о пространстве задачи и движении в нем, предполагают, что не существуют специфических механизмов для решения инсайтных задач: само продвижение по полю задачи осуществляется через специальные эвристики (Chronicle et al., 2001). Так, этот подход отрицает различия между инсайтными и неинсайтными задачами. Концепции специфического подхода, который в свою очередь является развитием идей гештальтпсихологов, свидетельствуют о различиях в механизмах решения инсайтных и неинсайтных задач. Так, С. Ольссон (Ohlsson, 1992) предполагает, что инсайт - выход из своеобразного тупика в процессе решения, вызванного сильным влиянием изначальной (и часто неверной) репрезентацией задачи. Тупик - это состояние, в котором невозможно дальше продвигаться в пространстве задачи. Для того чтобы преодолеть тупиковое состояние и продвинуться к верному решению, необходимо изменить представления о задаче, т.е. сменить репрезентацию. Наиболее часто описываются два механизма: а) ослабление ограничений - преодоление некоторых установок, запретов, которые накладываются самим субъектом; б) перекодирование - пересмотр состояния, условий задачи, ее переформулировка. К этому механизму можно отнести и декомпозицию чанков. Чанк - объединение решателем части материала в осмысленные совокупности. При столкновении с незнакомой проблемой происходит попытка сопоставить имеющийся набор элементов с предъявленными, если попытка оказывается неудачной - возникает тупиковая ситуация. Выходом из нее будет разложение (декомпозиция) «комплексных» элементов на более простые. Типичным примером может служить задача со спичками (Knoblich et al., 1999), в которой необходимо переместить только одну спичку для получения верного равенства: «XI = III + III». Чанком здесь 
выступает элемент «Х», который при изменении положения (т.е. декомпозиции) одной из его составляющих превращается в элемент «V».

На данный момент известно достаточно малое количество работ, в которых исследование инсайта осуществляется с использованием положений воплощенного подхода. Так, Л. Томас и А. Льерас (Thomas, Lleras, 2009b) на материале задачи Н. Майера на связывание двух веревок рассмотрели, влияет ли вид моторной активности, выполняемой в перерывах между попытками решения, на успешность решения самой задачи. Результаты показали, что испытуемые из релевантной группы (моторная активность соответствует принципу качания маятника) успешнее справляются с решением, чем испытуемые из нерелевантной группы (моторная активность не соответствует принципу маятника). Авторы утверждают, что полученный ими эффект влияния на успешность решения сильнее, чем тот же эффект, базирующийся на материале подсказок экспериментатора, описанный в оригинальной работе Н. Майера.

В качестве одной из серий собственного эксперимента данная работа была воспроизведена К. Вернер и М. Рааб (Werner, Raab, 2013). Исследователи добавили еще одну экспериментальную группу с моторной активностью, которая соотносится с новым принципом решения - занятием более высокой позиции. В результате выбор испытуемым способа решения соответствовал типу выполняемой моторной активности, что наблюдается и на материале неинсайтных задач в других исследованиях К. Вернер и М. Рааб.

Особый вклад в изучение механизмов инсайта на данный момент вносит особенная технология регистрации движения глаз. Это обусловлено тем, что решение инсайтных задач связано со сложностью в отслеживании самого протекания решения и вербализации этого процесса. Как один из вариантов преодоления существующей проблемы был предложен eye-tracking. Внедрение нового метода позволило не только собрать результаты, которые подтверждают ранние теоретические изыскания, например, о переструктурировании задачи через изменение формата ее репрезентации как основном механизме инсайтного решения (Владимиров, Чистопольская, 2016), но и расширить ВП, а именно изучать, каким образом моторная активность глаз влияет на процесс решения мыслительных задач. Отдельно стоит отметить работы, которые использовали задачу, разработанную К. Дункером: испытуемым необходимо было найти способ уничтожения опухоли желудка лучами лазера, который при определенной мощности способен разрушать органические ткани, но при этом здоровые части тела не должны быть затронуты. Схематически выделялась область опухли, здоровые ткани, внешняя граница кожи. Верным решением в данных условиях будет конвергенция нескольких лазеров слабой мощности на опухоли. Так, например (Grant, Spivey, 2003), было показано, что более успешные решатели задействовали больше саккадических движений глаз, пересекающих образно предоставленную «кожу». Это положение было подтверждено в исследованиях Л. Томас и А. Льерас (Thomas, Lleras, 2007, 2009a), а также группы китайских ученых (Xing et al., 2018). Причем результаты при условиях принудительного смещения за «кожу» (увеличение числа саккад) или фиксации на «опухоль» (уменьшение 
числа саккад) зрительного внимания позволили сделать вывод, что и специально направляемая экспериментатором двигательная активность глаз может оказывать существенное влияние на успешность решения задачи.

Таким образом, в области изучения процесса решения мыслительных задач в целом и для изучения феномена инсайта в частности для ВП есть большой и пока что не раскрытый потенциал. Мы предполагаем, что применение основных принципов воплощенного познания можно рассматривать как потенциальное подтверждение концепций специфического подхода: выполнение определенных действий, например выполнение простого моторного задания, может фасилитировать запуск одного из механизмов смены репрезентации. Целью нашего исследования является выявление роли моторной активности, содержащей и не содержащей в своей структуре основные принципы решения инсайтной задачи на симметрию. Мы предполагаем, что осуществляемая испытуемым двигательная активность влияет на процесс решения инсайтных задач: число испытуемых, успешно справившихся с заданием, будет наибольшим в группе, где моторная активность полностью воплощает принципы решения задачи.

\section{Участники исследования}

В исследовании приняло участие 45 испытуемых разного возраста и пола (20 женщин, 25 мужчин; средний возраст - 20.5 лет). Из них были сформированы три экспериментальные группы моторной активности в зависимости от того, насколько она отражает основные принципы решения задачи. Никто из испытуемых ранее не был знаком с задачей или ее решением.

\section{Стимульный материал и процедура}

В качестве материала, на основе которого рассматривалось влияние моторной активности на процесс решения инсайтных задач, была взята задача на симметрию (рисунок 1) (Чистопольская, Владимиров, 2017). Она представляет собой вертикальную последовательность фигур, которые образуются при зеркальном отражении чисел 1, 2, 3. Перед испытуемым ставится задача: обнаружить закономерность, которая заложена в последовательности этих трех фигур, и нарисовать следующую фигуру, которая соответствует этой закономерности, т.е. в ответе должна быть зеркально отражено число 4. Задача предоставлялась испытуемым в печатном виде.

\section{Инсайтная задача на симметрию}

Рисунок 1

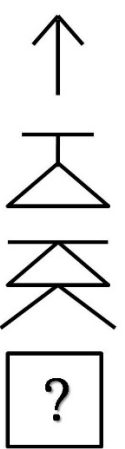


Эта задача имеет два основных принципа решения, без которых невозможно нахождение правильного ответа:

- Приниип декомпозиции чанка. Каждая из фигур является составной, ее элементами являются цифры: для успешного решения необходимо разбить эту «целостную» и большую фигуру на более простые и мелкие составляющие.

- Приниип симметрии. Каждая из этих фигур образуется путем зеркального отражения числа.

Мы выделили три группы моторной активности, которые представляют собой различные воплощения принципов решения задачи (см. рисунок 2).

1. Воплощенная группа: моторная активность в этой группе состояла в разрезании симметрично сложенного листа бумаги и воплощала и принцип декомпозиции чанка, и принцип симметрии - основные принципы решения данной задачи.

2. Полувоплощенная группа: моторная активность в этой группе состояла в разрезании асимметрично сложенного листа бумаги и воплощала лишь принцип декомпозиции чанка как основной принцип решения задачи.

3. Невоплощенная, или контрольная, группа: моторная активность в этой группе состояла в комканье листа бумаги и не воплощала необходимых принципов решения задачи.

Мы предполагали, что моторная активность с различным воплощением принципов решения задачи будет по-разному влиять на процесс решения, а наибольшее число решивших будет в группе, где моторная активность воплощает оба принципа решения задач.

Независимая переменная - тип моторной активности:

- воплощенная;

• полувоплощенная;

• контрольная.

Зависимая переменная - успешность решения задачи:

- время решения;

- правильность решения.

Процедура исследования. Непосредственно перед началом эксперимента испытуемым в зависимости от их моторной группы предлагалось разрезать или скомкать бумажный лист. Затем испытуемого знакомили с условием задачи,

Рисунок 2

Различные типы моторной активности: 1 - воплощенный, 2 - полувоплощенный, 3 - контрольный

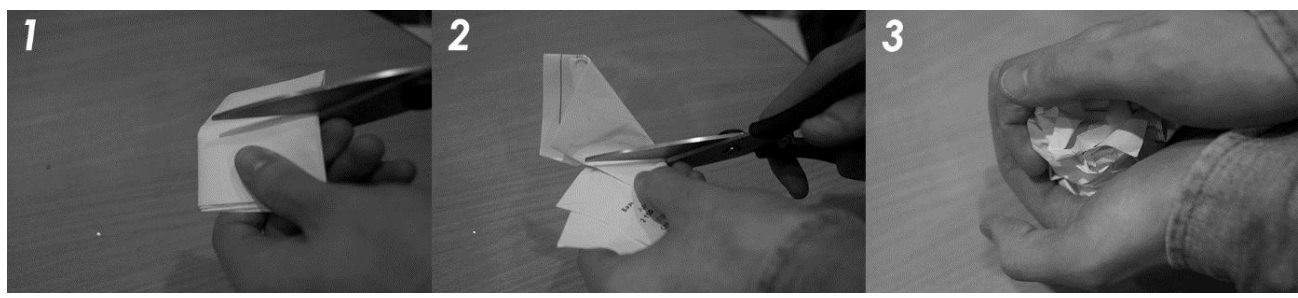


после чего он мог приступать к работе. Максимальное время решения для испытуемого составляло 40 минут и было разделено на 8 блоков по 5 минут каждый. После завершения одного блока испытуемого просили отвлечься от решения задачи и совершить те же манипуляции с листом бумаги, что и в начале эксперимента, после чего он мог снова возвращаться к решению. Фиксировались время и успешность решения. Результаты испытуемых, которые решили задачу, уложившись в восемь 5-минутных блоков, считались успешными. Успешно решивших задачу испытуемых опрашивали: заметили ли они связь между решением задачи и тем заданием, которое они выполняли в перерывах.

\section{Обработка результатов}

40\% испытуемых воплощенной группы справились с решением задачи, в то время как в контрольной группе только 6.7 \% испытуемых сумели найти решение в отведенное время. Для полувоплощенной группы этот показатель составил 33\%. Мы рассматривали два аспекта: время решения (рисунок 3) и его успешность (рисунок 4). Время решения задачи в группе воплощенной моторной активности меньше, чем в группе полувоплощенной моторной активности $(\mathrm{U}=1, p=0.01)$. Также в группе воплощенной моторной активности наблюдается большее число успешных решателей, чем в группе с контрольной моторной активностью $\left(\chi^{2}=4.658 ; p=0.03\right)$. Между группами полувоплощенной и контрольной моторной активности не обнаружено значимых различий $\left(\chi^{2}=3.333 ; p=0.06\right)$.

\section{Интерпретация результатов}

Группы воплощенной и полувоплощенной моторной активности различаются по времени решения, но не по количеству решивших. В обоих видах

Рисунок 3

График зависимости времени решения от типа моторной активности

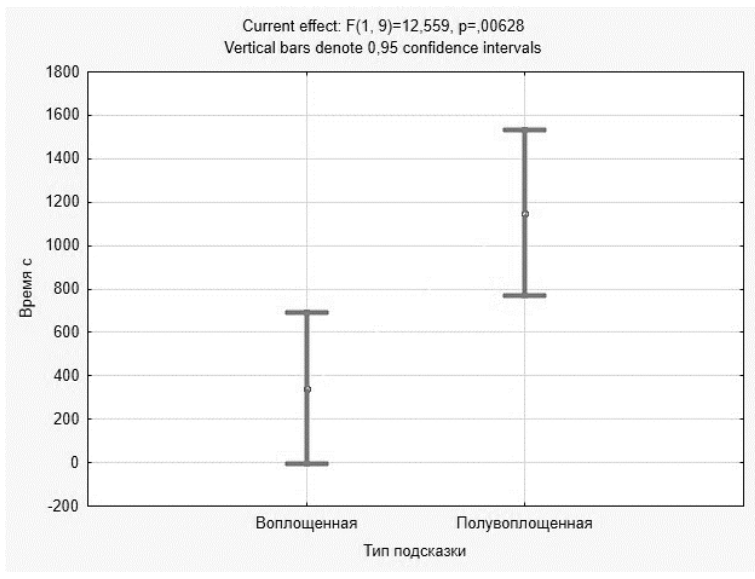


Рисунок 4

Распределение испытуемых по успешности решения в зависимости от типа моторной активности

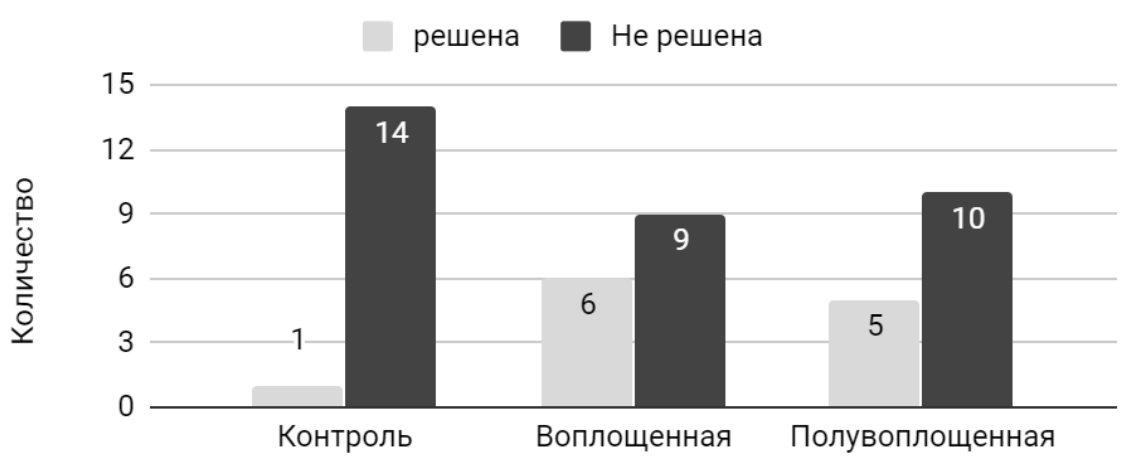

Тип моторной активности

активности был заложен принцип декомпозиции и только в воплощенной принцип симметрии. Таким образом, можно предположить, что большей значимостью для решения задач подобного типа обладает принцип декомпозиции чанка. В целом принцип симметрии мы можем рассматривать как уточнение к принципу декомпозиции: разделение должно быть исключительно таким, чтобы в результате получились две симметричные фигуры. Возможно, содержание обоих принципов решения в предварительной моторной активности ускоряет схватывание принципов симметрии и декомпозиции. Для принципа симметрии характерна одна особенность: 3 испытуемых (2 из воплощенной группы, 1 - из полувоплощенной) уловили симметричность фигур (сложили лист с задачей пополам или провели линию через середины всех трех фигур), однако так и не обнаружили, что отражены именно числа, и, таким образом, не справились с задачей в установленное время. Учитывая перечисленные особенности, можно модифицировать стимульный материал для создания отдельных условий для обоих принципов, а именно рассмотрение принципа симметрии вне его возможной связи с принципом декомпозиции.

Можно предположить возможную структуру взаимодействия моторной активности и нахождения верного решения инсайтной задачи. Если моторная активность содержит в себе те же элементы, что и механизмы смены репрезентации (или их более конкретизированные версии в виде принципов решения), то выполнение такой моторной операции приведет к имплицитной активации соответствующего механизма смены репрезентации. В таком случае для каждой из инсайтных задач, где необходимо непосредственное активное моторное взаимодействие (задача Н. Майера на связывание двух веревок, задачи К. Дункера и пр.), существует отличная от остальных моторная операция. Прочая же активность/положение тела не будет давать схожего положительного эффекта. Для проверки этого утверждения потребуется провести дополнительное исследование с различными типами инсайтных задач. 
Также в ходе выполнения задания испытуемые часто обращали внимание на то, как именно они выполняют моторную активность. Это проявлялось в вопросах экспериментатору: «Правильно ли я разрезаю лист?», «Я должен пересчитать получившиеся кусочки?», «Это связано с тем заданием?» и пр. Можно предположить, что подобный метакогнитивный компонент мог также повлиять на процесс решения: если испытуемые сознательно схватывали принцип декомпозиции через связь разрезания и того, что им нужно отыскать решение задачи, то в таком случае сама моторная активность не несет в себе никакого значимого для формирования инсайтного решения эффекта. В таком случае необходима конспирация моторной активности в таком же формате, который предложили Л. Томас и А. Льерас (Thomas, Lleras, 2009b) в своем исследовании: испытуемым рассказывается так называемая легенда, что на самом деле в данном эксперименте исследуется влияние выброса кислорода в кровь во время перерывов от решения задачи. В таком случае испытуемым нужно выполнять какое-либо моторное задание без попытки сознательного связывания выполняемого задания и условия истинной задачи.

Кроме конспирации, предлагается также сменить формат самой моторной активности: если в данном эксперименте испытуемый манипулировал искусственными объектами (ножницы, бумага), то в следующем исследовании выполнение моторного задания будет без использования так называемых артефактов среды. Нами проведено пилотажное исследование, в котором посредством метода многомерного шкалирования $(\mathrm{n}=22,13$ мужчин, средний возраст $=24.36$ ) определены три вида моторной активности, два из которых содержат принципы решения задачи на симметрию, а один, контрольный, который нужен для дополнительной проверки и не содержит ни одного принципа решения. Во всех случаях испытуемый совершает различные «разрывательные» или «симметричные» движения руками.

Необходимость дополнительного исследования заключается не только в смене формата моторной активности и исключении метакогнитивного компонента: для многих исследований в подходе ВП свойственна невоспроизводимость эффектов при полной репликации исследования. Так, результаты уже упоминавшегося нами исследования Мишо с соавт. (Michaux et al., 2013) не были воспроизведены в одном исследовании (Morrissey et al., 2020), которое одновременно являлось репликацией двух работ относительно пальцевого счета и его влияния на ментальную арифметику. Второй воспроизводимой работой было исследование (Newman, Soylu, 2014), в котором демонстрировалось, что люди, привыкшие считать начиная с правой руки, гораздо быстрее выбирают правильный ответ, чем те, кто начинают считать с левой руки (все испытуемые были правшами). В дополнение ко всему, исследование также было кросс-культурным и не показало таких различий в группе испытуемыхкитайцев. Эффективность и актуальность дальнейшего развития ВП напрямую зависит от того, насколько будут воспроизводимы эффекты, полученные ранее.

Итак, в результате исследования установлена фасилитирующая роль моторной активности в инсайтном решении задачи на симметрию. Запланированные модификации экспериментального плана позволят получить 
более достоверные и качественные результаты. Кроме того, планируется разработка таких типов моторной активности, которые можно было бы внедрить в решение других задач инсайтного типа.

\section{Литература}

Владимиров, И. Ю., Чистопольская, А. В. (2016). Анализ гностических действий с помощью технологии регистрации движения глаз как метод изучения процесса инсайтного решения. Культурно-историческая психология, 12(1), 24-34.

Лакофф, Д., Джонсон, М. (2004). Метафоры, которыми мы живем (2-е изд.). М.: Едиториал УРСС.

Логинов, Н. И., Спиридонов, В. Ф. (2017, а). Воплощенное познание (Embodied Cognition): основные направления исследований. Вестник СПбГУ. Психология и педагогика, 7(4), 343-364.

Логинов, Н. И., Спиридонов, В. Ф. (2017, б). Воплощенное познание как современный тренд развития когнитивной психологии. Вестник СПбГУ. Психология и педагогика, 7(1), 25-42.

Пономарев, Я. А. (1976). Психология творчества. М.: Наука.

Спиридонов, В. Ф. (2006). Психология мышиения: Решение задач и проблем: Учебное пособие. М.: Генезис.

Уилсон, М. (2012). Шесть взглядов на воплощенное познание. В кн. В. Ф. Спиридонов, М. В. Фаликман (ред.), Горизонты когнитивной психологии: хрестоматия (с. 19-28). М.: Российский государственный гуманитарный университет/Языки славянских культур.

Чистопольская, А. В., Лазарева, Н. Ю., Маркина, П. Н., Владимиров, И. Ю. (2019). Представление о высокоуровневых и низкоуровневых процессах в когнитивной психологии. Теория изменения репрезентации С. Ольссона с позиции уровневого подхода. Вестник ЯрГУ. Серия Гуманитарные науки, 3, 94-101.

Чистопольская, А. В., Владимиров, И. Ю., Секурцева, Ю. Г. (2017). Изменение репрезентации в процессе решения визуальных инсайтных задач. Вестник ЯрГУ. Серия Гуманитарные науки, $1,95-101$.

Ссылки на зарубежные источники см. в разделе References после англоязычного блока.

Курицын Александр Александрович - помощник исследователя, ЯрГУ им. П.Г. Демидова.

Сфера научных интересов: процессы решения мыслительных задач, инсайт, воплощенное познание, воплощенные метафоры.

Контакты: alexanderalkuritsyn@gmail.com

Чистопольская Александра Валерьевна - доцент, кафедра общей психологии, ЯрГУ им. П.Г. Демидова, кандидат психологических наук.

Сфера научных интересов: процессы решения мыслительных задач, инсайт, воплощенное познание.

Контакты: chistosasha@mail.ru 


\title{
The Role of Motor Component in Insight Problem Solving
}

\author{
A.A. Kuritsyn ${ }^{a}$, A.V. Chistopolskaya ${ }^{a}$ \\ ${ }^{a}$ P.G. Demidov Yaroslavl State University, 14 Sovetskaya Str., Yaroslavl, 150000, Russian Federation
}

\begin{abstract}
In cognitive psychology, the ideas of body and motor influence on mental processes are quite relevant. The set of these ideas forms a new direction in research - embodied cognition research program. However, despite the wide prevalence, only a small number of studies apply principles of this approach to study the phenomenon of insight. At present, there are several theories that try to explain mechanisms of insight problem solving in different ways: nonspecific approach, which denies differences in processes of solving insight and non-insight problems, and specific approach, which implies special mechanisms for insight problem solving. Our study, based on the ideas of embodied cognition research program and specific approach to understanding insight, examines insight problem solving depending on motor activity, which contains different types of principles of solving insight symmetry task: the principle of symmetry and the principle of chunk decomposition. Three experimental groups of motor activity were formed, which embody both principles of problem solving in different ways. Differences were found in the time needed for solving problems depending on associated type of motor activity. We found the facilitative effect of motor activity, which embodies both basic principles of insight problem solving. It is assumed that the presence of the problem solving principles in motor activity implicitly triggers the mechanisms of representation change. Future research plans are outlined to test the principles of solving insight task based on non-instrumental motor activity. The results are consistent with Ponomarev's ideas on the role of a by-product in thought processes.
\end{abstract}

Keywords: insight, motor activity, embodied cognition, chunk decomposition, symmetry insight problem.

\section{References}

Chistopolskaya, A. V., Lazareva, N. Yu., Markina, P. N., \& Vladimirov, I. Yu. (2019). The concept of high-level and low-level processes in cognitive psychology. S. Olsson's representational change theory from the position of the level approach. Vestnik Yaroslavskogo Gosudarstvennogo Universiteta im. P. G. Demidova. Seriya Gumanitarnye Nauki, 3, 94-101. (in Russian)

Chistopolskaya, A. V., Vladimirov, I. Yu., \& Sekurtseva, J. G. (2017). Change of representation in the process of visual insight problem-solving. Vestnik Yaroslavskogo Gosudarstvennogo Universiteta im. P. G. Demidova. Seriya Gumanitarnye Nauki, 1, 95-101. (in Russian)

Chronicle, E. P., Ormerod, T. C., \& MacGregor, J. N. (2001). When insight just won't come: The failure of visual cues in the nine-dot problem. The Quarterly Journal of Experimental Psychology Section A: Human Experimental Psychology, 54(3), 903-919. doi:10.1080/713755996 
Dudschig, C., de la Vega, I., \& Kaup, B. (2014). Embodiment and second-language: Automatic activation of motor responses during processing spatially associated L2 words and emotion L2 words in a vertical Stroop paradigm. Brain and Language, 132, 14-21. doi:10.1016/j.bandl.2014.02.002

Glenberg, A. M. (2010). Embodiment as a unifying perspective for psychology. Wiley Interdisciplinary Revieres: Cognitive Science, 1(4), 586-596. doi:10.1002/wcs.55

Grant, E. R., \& Spivey, M. J. (2003). Eye movements and problem solving: Guiding attention guides thought. Psychological Science, 14(5), 462-466. doi:10.1111/1467-9280.02454

Knoblich, G., Ohlsson, S., Haider, H., \& Rhenius, D. (1999). Constraint relaxation and chunk decomposition in insight problem solving. Journal of Experimental Psychology: Learning, Memory, and Cognition, 25(6), 1534-1555. doi:10.1037/0278-7393.25.6.1534

Lakoff, G., \& Johnson, M. (2004). Metafory, kotorymi my zhivem [Metaphors we live by] (2nd ed.). Moscow: Editorial URSS. (in Russian; transl. of: Lakoff, G., \& Johnson, M. (1980). Metaphors we live by. Chicago: University of Chicago Press.)

Loginov, N. I., \& Spiridonov, V. F. (2017, a). Key areas of research in the embodied cognition approach. Vestnik Sankt-Peterburgskogo Universiteta. Psikhologiya i Pedagogika, 7(4), 343-364. (in Russian)

Loginov, N. I., \& Spiridonov, V. F. (2017, b). Embodied cognition as a current trend in cognitive psychology. Vestnik Sankt-Peterburgskogo Universiteta. Psikhologiya i Pedagogika, 7(1), 25-42. (in Russian)

Michaux, N., Masson, N., Pesenti, M., \& Andres, M. (2013). Selective interference of finger movements on basic addition and subtraction problem solving. Experimental Psychology, 3(60), 197-205. doi:10.1027/1618-3169/a000188

Morrissey, K., Hallett, D., Wynes, R., Kang, J., \& Han M. (2020). Finger-counting habits, not finger movements, predict simple arithmetic problem solving. Psychological Research, 84(1), 140-151. doi:10.1007/s00426-018-0990-y

Newell, A., \& Simon, H. A. (1972). Human problem solving. Englewood Cliffs, NJ: Prentice-Hall.

Newman, S. D., \& Soylu, F. (2014). The impact of finger counting habits on arithmetic in adults and children. Psychological Research, 78(4), 549-556. doi:10.1007/s00426-013-0505-9

Ohlsson, S. (1992). Information-processing explanations of insight and related phenomena. In M. T. Keane \& K. J. Gilhooly (Eds.), Advances in the psychology of thinking (pp. 1-44). New York: Harvester Wheatsheaf.

Ponomarev, Ya. A. (1976). Psikhologiya tvorchestva [The psychology of creativity]. Moscow: Nauka. (in Russian)

Spiridonov, V. F. (2006). Psikhologiya myshleniya: Reshenie zadach i problem [The psychology of thinking: Solving tasks and problems]. Moscow: Genezis. (in Russian)

Thomas, L. E., \& Lleras, A. (2007). Moving eyes and moving thought: On the spatial compatibility between eye movements and cognition. Psychonomic Bulletin and Reviere, 14(4), 663-668. doi:10.1167/6.6.871

Thomas, L. E., \& Lleras, A. (2009, a). Covert shifts of attention function as an implicit aid to insight. Cognition, 111, 168-174. doi:10.1016/j.cognition.2009.01.005

Thomas, L. E., \& Lleras, A. (2009, b). Swinging into thought: directed movement guides insight in problem solving. Psychonomic Bulletin and Review, 16(4), 719-723. doi:10.3758/PBR.16.4.719

Vladimirov, I. Yu., \& Chistopolskaya, A. V. (2016). Analysis of gnostic actions using eye tracking as a method of studying insight in problem solving. Kul'turno-istoricheskaya Psikhologiya [CulturalHistorical Psychology], 12(1), 24-34. doi:10.17759/chp.2016120103. (in Russian)

Werner, K., \& Raab, M. (2013). Moving to solution effects of movement priming on problem solving. Experimental Psychology, 60(6), 403-409. doi:10.1027/1618-3169/a000213 
Werner, K., \& Raab, M. (2014). Moving your eyes to solution: effects of movements on the perception of a problem-solving task. The Quarterly Journal of Experimental Psychology, 67(8), 1571-1578. doi:10.1080/17470218.2014.889723

Werner, K., Raab, M., \& Fischer, M. H. (2019). Moving arms: the effects of sensorimotor information on the problem-solving process. Thinking and Reasoning, 25(2), 171-191. doi:10.1080/13546783.2018.1494630

Wilson, M. (2012). Shest' vzglyadov na voploshchennoe poznanie [Six views of embodied cognition]. In V. F. Spiridonov \& M. V. Falikman (Eds.), Gorizonty kognitivnoi psikhologii [Horizons of cognitive psychology] (pp. 19-28). Moscow: Rossiiskii gosudarstvennyi gumanitarnyi universitet/Yazyki slavyanskikh kul'tur. (in Russian; transl. of: Wilson, M. (2002). Six views of embodied cognition. Psychonomic Bulletin and Review, 9, 625-636.)

Xing, Q., Rong, C., Lu, Zh., Yao, Y., Zhang, Zh., \& Zhao, X. (2018). The effect of the embodied guidance in the insight problem solving: An eye movement study. Frontiers in Psychology, 9, 1-14. doi:10.3389/fpsyg.2018.02257

Alexander A. Kuritsyn - Research Assistant, P.G. Demidov Yaroslavl State University. Research Area: insight problem solving, insight, embodied cognition, embodied metaphors. E-mail: alexanderalkuritsyn@gmail.com

Alexandra V. Chistopolskaya - Associate Professor, P.G. Demidov Yaroslavl State University, $\mathrm{PhD}$ in Psychology.

Research Area: Insight problem solving, insight, embodied cognition.

E-mail: chistosasha@mail.ru 